\title{
Evaluation of Approaches for Tracking Virus Particles in Fluorescence Microscopy Images
}

\author{
W. J. Godinez ${ }^{1}$, M. Lampe ${ }^{2}$, S. Wörz ${ }^{1}$, B. Müller ${ }^{2}$, R. Eils ${ }^{1}$, K. Rohr ${ }^{1}$ \\ ${ }^{1}$ University of Heidelberg, BIOQUANT, IPMB, and DKFZ Heidelberg, \\ Dept. Bioinformatics and Functional Genomics, Biomedical Computer Vision Group, \\ Im Neuenheimer Feld 267, 69120 Heidelberg, Germany \\ ${ }^{2}$ University of Heidelberg, Dept. of Virology, \\ Im Neuenheimer Feld 324, 69120 Heidelberg, Germany \\ wgodinez@ieee.org
}

\begin{abstract}
Tracking virus particles in fluorescence microscopy image sequences enables the characterization of the dynamical behavior of these objects. Several approaches have been developed for the task of virus tracking. However, few studies have quantitatively evaluated the performance of the different approaches. Such a comparison is essential to predict the performance of the approaches under realistic conditions. In this paper, we present a quantitative evaluation of eight approaches for tracking virus particles. We have investigated deterministic and probabilistic approaches. The evaluation is based on nine real microscopy image sequences of virus particles, for which ground truth was obtained by manual tracking.
\end{abstract}

\section{Introduction}

Tracking single virus particles in fluorescence time-lapse microscopy images yields quantitative information that describes their dynamical behavior. Such information can be employed to characterize the influence of antiviral drugs. To obtain statistically sound conclusions, a large number of particles must be tracked. Therefore, automatic tracking approaches are required to efficiently handle the large amount of image data.

Several approaches for virus tracking have been described. Typically, deterministic approaches have been employed (e.g., [1, 2]). More recently, probabilistic approaches (e.g., $[3,4]$ ) have been introduced. However, few studies have quantitatively compared the performance of virus tracking approaches. Such a comparison is needed to predict the performance of the approaches for real images. The most detailed comparison of tracking approaches for fluorescent particles has been presented in [5]. There, the authors evaluate the performance of four deterministic tracking approaches using synthetic images with a focus on object localization. One main finding is that the performance of the tracking approaches declines as the signal-to-noise ratio (SNR) decreases. While the study is relatively detailed, it has three shortcomings. First, the performance measure is based on the localization error, and the influence of errors 
in correspondence finding is ignored. Second, no real images have been used. Third, only deterministic approaches are considered.

In this work, we present a quantitative performance evaluation of approaches for tracking multiple virus particles in microscopy image sequences. In total, we have evaluated eight tracking approaches. We have analyzed two deterministic approaches as well as six probabilistic approaches. The deterministic approaches are based on either the spot-enhancing filter [6] or 2D Gaussian fitting for particle localization, and a global nearest neighbor approach for motion correspondence. The probabilistic approaches are based on Kalman filters, mixture of particle filters (MPF), and independent particle filters (IPF). The approaches have been applied to synthetic image sequences displaying virus-like objects, as well as to 9 different real microscopy image sequences (each comprising between 150 and 400 frames) displaying HIV-1 particles. In comparison to [5], the employed performance measure reflects more comprehensively the performance of the evaluated tracking approaches.

\section{Materials and methods}

\subsection{Deterministic tracking approaches}

The deterministic approaches follow a two-step paradigm consisting of object localization and motion correspondence. For object localization, we employed two approaches: an approach based on the spot-enhancing filter (SEF), and an approach using 2D Gaussian fitting (GaussFit). For motion correspondence, we used a global nearest neighbor (GNN) approach [2]. By combining the localization schemes with the motion correspondence scheme we obtain two deterministic approaches: 1) spot-enhancing filter with global nearest neighbor (SEF\&GNN), and 2) 2D Gaussian fitting with global nearest neighbor (GaussFit\&GNN).

\subsection{Probabilistic tracking approaches}

The probabilistic approaches follow a Bayesian paradigm, where the aim is to estimate the state $\mathbf{x}_{t}$ of a virus particle at time step $t$ given a sequence of measurements $\mathbf{y}_{1: t}$. A solution to this problem involves computing the posterior distribution $p\left(\mathbf{x}_{t} \mid \mathbf{y}_{1: t}\right)$ using stochastic propagation and Bayes' theorem:

$$
p\left(\mathbf{x}_{t} \mid \mathbf{y}_{1: t}\right) \propto p\left(\mathbf{y}_{t} \mid \mathbf{x}_{t}\right) p\left(\mathbf{x}_{t} \mid \mathbf{y}_{1: t-1}\right) .
$$

Given certain assumptions on the form of the distributions, the recursion can be solved analytically using a Kalman filter. More generally, the recursive relation can be solved via approximation using a particle filter. The idea behind this filter is to approximate the posterior distribution using a set of weighted random samples. When tracking multiple objects, multiple modes arise in the posterior distribution. The multimodality can be modeled via a non-parametric $M$-component mixture model, which can be computed using a mixture of particle filters [7]. Alternatively, one may track multiple objects by instantiating one 
independent particle filter per object (e.g., [8]). To prevent the problem of filter coalescence, the IPF includes a penalization scheme [4]. Similarly, one may use one Kalman filter per object to track multiple objects.

For all probabilistic approaches, we employed the two localization schemes described above to detect virus particles. Note that the approaches using a mixture of particle filters can only track a fixed number of objects. For the approaches using independent particle filters and Kalman filters, the motion correspondence problem, i.e., the problem of assigning the position measurements computed by the localization schemes to each spatial-temporal filter, is addressed using a global nearest neighbor approach. The combination of the two localization algorithms with the different filters yields the following tracking approaches: 3 ) spot-enhancing filter and Kalman filters (SEF\&Kalman), 4) spot-enhancing filter and a mixture of particle filters (SEF\&MPF), 5) spot-enhancing filter and independent particle filters (SEF\&IPF), 6) 2D Gaussian fitting and Kalman filters (GaussFit\&Kalman), 7) 2D Gaussian fitting and a mixture of particle filters (GaussFit\&MPF), and 8) 2D Gaussian fitting and independent particle filters (GaussFit\&IPF).

\subsection{Performance assessment}

To quantitatively assess the performance of each approach in each image sequence, we have employed the tracking accuracy $P_{\text {track }}=\frac{n_{\text {track,correct }}}{n_{\text {track,total }}}$, which reflects the ratio between the number of correctly computed trajectories $n_{\text {track,correct }}$ and the number of true trajectories $n_{\text {track,total }}$. The value $n_{\text {track,correct }}$ is computed as the weighted sum of the percentage of tracked time steps $r_{\text {tracked, } i}$ for each $i$-th true trajectory: $n_{\text {track,correct }}=\sum_{i=1}^{n_{\text {track,total }}} w_{i} r_{\text {tracked, } i}$, where the weight $w_{i}$ is given by a Gaussian function $G(\cdot)$, which takes as its argument the number of correctly computed trajectories $n_{\text {track,i }}$ corresponding to each $i$-th true trajectory: $w_{i}=G\left(n_{\text {track }, i} ; \mu=1, \sigma=1\right)$. The weighting scheme is introduced to penalize computed trajectories that are broken. A computed trajectory is assumed to be correct if the Euclidean distance between the measured object position and the true object position is below 2 pixels.

\section{Results}

We have applied all eight approaches to synthetic as well as real microscopy images. Below, we present the results for nine real microscopy image sequences. In these sequences, fluorescently labeled HIV-1 particles were imaged using a fluorescence wide-field microscope; movies were recorded with a frequency of $10 \mathrm{~Hz}$. Ground truth for the virus positions was obtained by manual tracking using the commercial software MetaMorph. For all sequences, we have employed fixed parameter values for all approaches. Similarly, the noise parameters for the dynamical model of the Kalman filter were set analogously as the ones employed for the particle filter. Details for each sequence are given in Table 1. The quantitative experimental results for the nine sequences are presented in Table 2. As an example, results for the real image sequence "Seq. 7" are shown in Fig. 1. 
Table 1. Description of real microscopy image sequences.

\begin{tabular}{lccc}
\hline & Dimensions [pixels] & No. of time steps & No. of objects \\
\hline Seq. 1 & $256 \times 256$ & 250 & 23 \\
Seq. 2 & $256 \times 256$ & 250 & 10 \\
Seq. 3 & $256 \times 256$ & 250 & 5 \\
Seq. 4 & $256 \times 256$ & 150 & 21 \\
Seq. 5 & $512 \times 512$ & 200 & 15 \\
Seq. 6 & $512 \times 512$ & 400 & 29 \\
Seq. 7 & $512 \times 512$ & 400 & 31 \\
Seq. 8 & $512 \times 512$ & 400 & 43 \\
Seq. 9 & $512 \times 512$ & 400 & 24 \\
\hline
\end{tabular}

Table 2. Tracking accuracy $P_{\text {track }}$ for real microscopy image sequences.

\begin{tabular}{lcccccccc}
\hline & \multicolumn{1}{c}{ SEF\& } & SEF\& & \multicolumn{2}{c}{ SEF\& } & SEF\& GaussFit\& GaussFit\& & GaussFit\& GaussFit\& \\
& GNN & Kalman & MPF & IPF & GNN & Kalman & MPF & IPF \\
\hline Seq. 1 & 75.24 & 81.12 & 84.82 & 86.73 & 71.29 & 81.30 & 81.95 & 82.61 \\
Seq. 2 & 60.78 & 68.70 & 71.93 & 83.52 & 30.79 & 44.64 & 63.49 & 84.67 \\
Seq. 3 & 45.07 & 32.29 & 76.67 & 42.55 & 40.69 & 60.18 & 60.00 & 80.00 \\
Seq. 4 & 61.71 & 68.89 & 28.52 & 71.10 & 70.53 & 74.59 & 23.81 & 83.39 \\
Seq. 5 & 93.54 & 93.54 & 84.64 & 93.54 & 81.09 & 93.54 & 85.64 & 93.54 \\
Seq. 6 & 57.79 & 74.59 & 39.64 & 63.98 & 55.18 & 67.97 & 41.73 & 62.10 \\
Seq. 7 & 64.65 & 80.60 & 48.26 & 82.48 & 53.21 & 77.47 & 31.23 & 77.67 \\
Seq. 8 & 74.61 & 74.09 & 50.62 & 74.64 & 75.10 & 74.11 & 49.04 & 67.76 \\
Seq. 9 & 72.55 & 82.55 & 48.51 & 73.55 & 67.78 & 80.55 & 52.61 & 76.89 \\
\hline Mean & 67.33 & 72.93 & 59.29 & 74.68 & 60.63 & 72.71 & 54.39 & 78.74 \\
Std. Dev. 13.68 & 17.08 & 20.62 & 15.00 & 16.82 & 13.99 & 20.96 & 9.32 \\
\hline
\end{tabular}

\section{Discussion}

Our experimental results in Table 2 indicate that the performance of the deterministic approaches is not very good (e.g., for the best deterministic approach, namely SEF\&GNN, we obtain a mean tracking accuracy of $\bar{P}_{\text {track }}=67.33 \%$ ). The reason for this is that localization errors (e.g., detection failures) as well as errors in correspondence finding (e.g, incorrect assignments) reduce the number of correctly computed trajectories. The approaches based on the Kalman filter, in comparison to the deterministic approaches, yield an improved performance (e.g., $\bar{P}_{\text {track }}=72.93 \%$ for the best Kalman-based approach, namely SEF\&Kalman). This suggests that the inclusion of a spatial-temporal filtering step enhances the performance. For the approaches using particle filters, it turned out that those using independent particle filters (IPF) outperform those using a mixture of particle filters (MPF). The reason for this result is twofold: 
Fig. 1. Tracking results for four approaches for the real image sequence "Seq. 7" (time step $t=110)$.

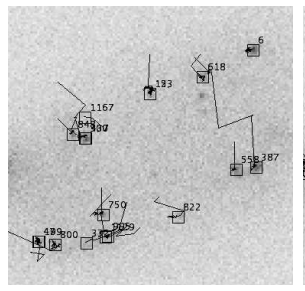

(a) SEF\&GNN

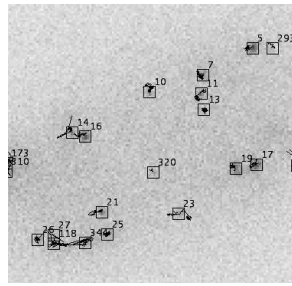

(b) SEF\&Kalman

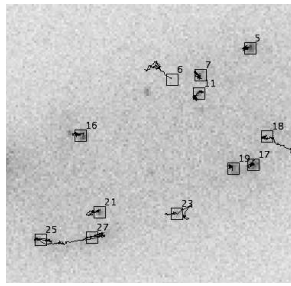

(c) SEF\&MPF

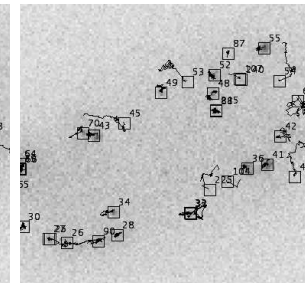

(d) SEF\&IPF

first, MPF cannot track a variable number of objects (whereas in the real images, the number of objects varies over time); second, the MPF uses a variable number of samples to estimate each mixture component. As such, the estimation accuracy decreases for those components for which few samples have been assigned. Among all approaches, the best tracking accuracy is obtained by GaussFit\&IPF $\left(\bar{P}_{\text {track }}=78.74 \%\right)$. The superior performance is mainly due to the comprehensive tracking machinery of the particle filter, which includes the steps of particle localization, motion correspondence, and position estimation. In summary, the quantitative results suggest that the probabilistic approaches are more accurate than the deterministic schemes.

Acknowledgement. Support of the BMBF (FORSYS) project VIROQUANT is gratefully acknowledged.

\section{References}

1. Schutz GJ, Schindler H, Schmidt T. Single-molecule microscopy on model membranes reveals anomalous diffusion. Biophys J. 1997;73(2):1073-1080.

2. Sbalzarini IF, Koumoutsakos P. Feature point tracking and trajectory analysis for video imaging in cell biology. J Struct Biol. 2005;151(2):182-195.

3. Arhel N, Genovesio A, Kim K, et al. Quantitative four-dimensional tracking of cytoplasmic and nuclear HIV-1 complexes. Nat Methods. 2006;3(10):817-824.

4. Godinez WJ, Lampe M, Wörz S, et al. Probabilistic tracking of virus particles in fluorescence microscopy images. Proc ISBI. 2008; p. 272-275.

5. Cheezum MK, Walker WF, Guilford WH. Quantitative comparison of algorithms for tracking single fluorescent particles. Biophys J. 2001;81:2378-2388.

6. Sage D, Neumann FR, Hediger F, et al. Automatic tracking of individual fluorescence particles: Application to the study of chromosome dynamics. IEEE Trans Image Process. 2005;14(9):1372-1382.

7. Vermaak J, Doucet A, Pérez P; IEEE. Maintaining multi-modality through mixture tracking. Proc ICCV. 2003; p. 1110-1116.

8. Qu W, Schonfeld D, Mohamed M; IEEE. Real-time interactively distributed multi-object tracking using a magnetic-inertia potential model. Proc IEEE ICCV. 2005;1:535-540. 